Assessment and Mitigation of

Diagnostic-Generated Electromagnetic Interference at the National Ignition Facility

C. G. Brown, M. J. Ayers, B. Felker, W. Ferguson, J. P. Holder, S. R. Nagel, K. W. Piston, N. Simanovskaia, A. L. Throop, M. Chung, T. Hilsabeck

April 24, 2012

High Temperature Plasma Diagnostics Monterey, CA, United States

May 6, 2012 through May 10, 2012 
This document was prepared as an account of work sponsored by an agency of the United States government. Neither the United States government nor Lawrence Livermore National Security, LLC, nor any of their employees makes any warranty, expressed or implied, or assumes any legal liability or responsibility for the accuracy, completeness, or usefulness of any information, apparatus, product, or process disclosed, or represents that its use would not infringe privately owned rights. Reference herein to any specific commercial product, process, or service by trade name, trademark, manufacturer, or otherwise does not necessarily constitute or imply its endorsement, recommendation, or favoring by the United States government or Lawrence Livermore National Security, LLC. The views and opinions of authors expressed herein do not necessarily state or reflect those of the United States government or Lawrence Livermore National Security, LLC, and shall not be used for advertising or product endorsement purposes. 


\title{
Assessment and Mitigation of Diagnostic-Generated Electromagnetic Interference at the National Ignition Facility ${ }^{\text {a) }}$
}

\author{
C. G. Brown Jr., ${ }^{1, b)}$ J. Ayers, ${ }^{1}$ B. Felker, ${ }^{1}$ W. Ferguson, ${ }^{1}$ J. P. Holder, ${ }^{1}$ S. R. Nagel, ${ }^{1}$ \\ K. W. Piston, ${ }^{1}$ N. Simanovskaia, ${ }^{1}$ A. L. Throop, ${ }^{1}$ M. Chung, ${ }^{2}$ T. Hilsabeck ${ }^{2}$ \\ ${ }^{1}$ Lawrence Livermore National Laboratory, Livermore, California, USA \\ ${ }^{2}$ General Atomics, San Diego, California, USA
}

\begin{abstract}
(Presented XXXXX; received XXXXX; accepted XXXXX; published online XXXXX)
Electromagnetic interference (EMI) is an ever-present challenge at laser facilities such as the National Ignition Facility (NIF). The major source of EMI at such facilities is laser-target interaction that can generate intense electromagnetic fields within, and outside of, the laser target chamber. In addition, the diagnostics themselves can be a source of EMI, even interfering with themselves. In this paper we describe EMI generated by ARIANE and DIXI, present measurements, and discuss effects of the diagnostic-generated EMI on ARIANE's CCD and on a PMT nearby DIXI. Finally we present some of the efforts we have made to mitigate the effects of diagnostic-generated EMI on NIF diagnostics.
\end{abstract}

\section{INTRODUCTION}

Electromagnetic interference (EMI) is an ever-present challenge at laser facilities such as the National Ignition Facility (NIF). The major source of EMI at such facilities is the lasertarget interaction that can generate intense electromagnetic fields within, and outside of, the laser target chamber. Such fields, called electromagnetic pulse (EMP), have been characterized in laser facilities ${ }^{1-3}$, including LLNL's $\operatorname{Titan}^{4}$ and Comet short-pulse lasers, as well as in $\mathrm{NIF}^{4}$.

However, EMP is not the only source of EMI at a laser facility. The diagnostics themselves can be a source of EMI, even interfering with the very diagnostic that generated the EMI. For example, pulsed-power circuits within a framing camera could generate electromagnetic fields that couple into sensitive electronics, such as Photo-multiplier Tubes (PMTs), of nearby diagnostics. Also, such pulsed-power generated electromagnetic fields could interfere with a Charge-Coupled Device (CCD) camera inside of the framing camera that generated the electromagnetic fields. Thus, understanding diagnostic-generated EMI and mitigating against it are important aspects of proper design and operation of diagnostics at NIF and other laser facilities.

In this paper we describe EMI generated by the Active Readout In A Neutron Environment ${ }^{5}$ (ARIANE) and DIlation Xray Imager $^{6}$ (DIXI) diagnostics and indicate possible effects of the diagnostic-generated EMI on ARIANE, DIXI, and the FilterFluorescer Experiment ${ }^{7}$ (FFLEX), another nearby diagnostic. Finally we present some of the efforts we have made to mitigate the effects of diagnostic-generated EMI on NIF diagnostics.

\footnotetext{
${ }^{\text {a) }}$ Contributed paper published as part of the Proceedings of the 19th Topical Conference on High-Temperature Plasma Diagnostics, Monterey, California, May, 2012.

${ }^{\text {b) }}$ Author to whom correspondence should be addressed: brown207@1lnl.gov.
}

\section{OVERVIEW OF SOURCES OF EMI}

Various sources of EMI are present in laser facilities. Some of the sources in NIF are represented in schematic form in Fig 1. These sources include fields from the laser-target interaction and diagnostic-generated EMI. Figure 1 also indicates possible EMI coupling paths to vulnerable electronics in diagnostics in the target chamber and the target bay. EMI from facility noise (pulsed power, motors, etc.) is not included here, but could be present also.

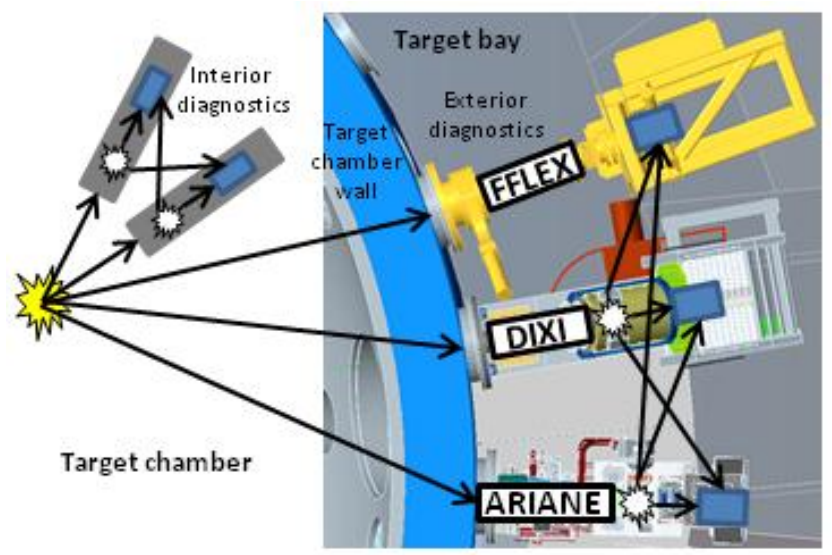

FIG. 1. (color online) Various sources of EMI in a portion of NIF, affecting the ARIANE, DIXI, and FFLEX diagnostics. The left-most burst symbol (yellow) represents the laser-target interaction. The other, smaller burst symbols (white) represent sources of diagnostic generated EMI. The laser-target interaction and diagnostic-generated EMI couple to vulnerable electronics (blue boxes) in FFLEX, DIXI, and ARIANE via EMI coupling paths, which are depicted as arrows. EMI from facility noise 
(pulsed power, motors, etc.) is not included here, but could be present also.

Examples of diagnostics that generate EMI are ARIANE and DIXI. ARIANE is located in NIF on the target bay side of the target chamber wall, and DIXI will be located near it (see Fig 1). Some of the sources of EMI in ARIANE include short, highvoltage pulses across the Micro Channel Plate (MCP) microstrips and phosphor, as well as fields generated by pulsed power and associated cables. See Fig 2. Some of the sources of EMI in DIXI include short, high-voltage pulses across the MCP and photocathode microstrips, as well as pulsed power and associated cables. DIXI also has electromagnets and associated pulsed power and cables that generate EMI. Refer to Fig 3. We will discuss EMI from ARIANE and DIXI in the following sections.

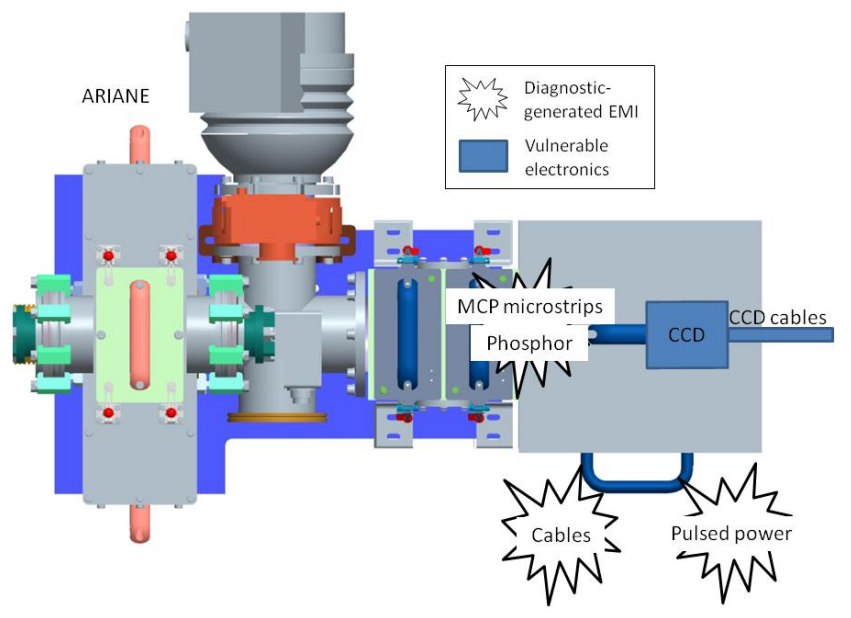

FIG. 2. (color online) Some of the sources of EMI in ARIANE.

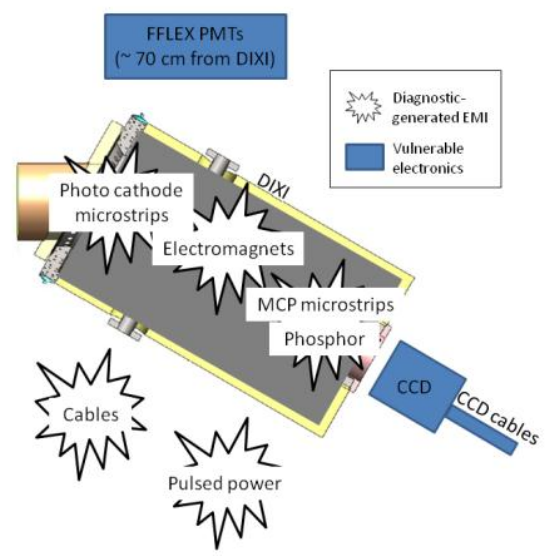

FIG. 3. (color online) Some of the sources of EMI in DIXI.

\section{EMI FROM ARIANE}

Before ARIANE was installed in NIF, electromagnetic field measurements were made while it was being pulsed in the laboratory. The measurements were taken using Prodyn RB-230 (high-frequency) and RB-270 (middle-frequency) B-dot sensors. For these measurements, the MCP microstrip and CCD camera area was not covered with an EMI box. Measurements indicated that the magnetic flux density had a maximum absolute value of $90 \mathrm{mG}$ on top of the CCD camera, corresponding to an electric field strength of $2.7 \mathrm{kV} / \mathrm{m}$ using the approximate relation $\mathrm{E} \approx \mathrm{cB}$, which is exact for plane waves. CCD camera lockups were noted in the laboratory testing.

When ARIANE was installed in NIF, the cables for the microstrip pulsed power and the CCD camera were dressed in braided shields, which were attached by mechanical compression at the ends to reduce EMI leakage. Also, the CCD was covered as well as possible with aluminum foil shielding. In order to isolate them from the ambient target bay fields, the microstrips, pulsed power, and CCD were covered in EMI enclosures, and the cables were placed in conduit. However, the front of the CCD still remained open the EMI from the microstrips, as it must to record light from the phosphor. When ARIANE was tested in NIF with the additional shielding, the CCD did not lock up, indicating that the EMI that caused the lock ups probably coupled into the CCD through the cables and not primarily through the front of the CCD. If the open front of the CCD becomes a problematic coupling path, transparent EMI films could be explored as mitigation.

\section{EMI FROM DIXI}

In light of experience with ARIANE, more measurements were taken of DIXI in the laboratory in order to design proper shielding earlier in the design cycle. Both low- and highfrequency magnetic field measurements were made with DIXI installed on the port of a short-pulse laser (laser not fired). The measurements were made while DIXI was pulsed in a realistic fashion. However, cables were not routed in conduit, as they will be in NIF. Further, the output of an FFLEX PMT was monitored while DIXI was pulsed, since FFLEX will be close to DIXI when DIXI is installed in NIF (see Fig 1).

The low-frequency $(5 \mathrm{~Hz}$ to $50 \mathrm{kHz}$ ) peak magnetic flux density measurements, made using a Lake Shore Model 475 DSP Gaussmeter set to peak measurement mode, indicated magnetic flux densities below $1 \mathrm{G}$ at $1 \mathrm{~m}$ from DIXI, except above DIXI where cables and a turbopump were located. These values are deemed acceptable, but a planned increase in solenoid current will likely bump the low-frequency magnetic flux densities above the current acceptable limit of $\sim 1 \mathrm{G}$ at $1 \mathrm{~m}$ from DIXI.

The high-frequency magnetic field measurements were made using the same B-dot sensors as in Section III. EMI FROM ARIANE. Measurements indicated a maximum absolute value of $23 \mathrm{mG} 1 \mathrm{~m}$ from DIXI, corresponding to an electric field strength of $0.7 \mathrm{kV} / \mathrm{m}$ using the approximate relation $\mathrm{E} \approx \mathrm{cB}$. Closer to the MCP microstrips, the fields are much higher: 47 to $333 \mathrm{mG}(1.4$ to $10 \mathrm{kV} / \mathrm{m}$ ), depending on which sensor was used for the measurement.

In order to determine what effect DIXI might have on the neighboring FFLEX diagnostic, a single PMT from FFLEX was set up in the laboratory at the same distance from DIXI that it will be when DIXI is installed in NIF. The output cable of the PMT was monitored. By turning on all of the pulses simultaneously (normal operation) and then pulsing each one individually, it was determined that the MCP pulse affected the 
PMT most, followed by the photo-cathode pulse. See Fig 4. The EMI coupling due to the MCP pulse alone was about twice as high as the FFLEX PMT signal level when in NIF $(\sim 50 \mathrm{mV})$, while the EMI coupling due to the photo-cathode pulse was about as high as the FFLEX PMT signal level. This is understandable because the FFLEX PMT was closer to the MCP than the photocathode, and the MCP microstrips were bare (unshielded) where they exit the CCD-end of DIXI creating antennas. In contrast, the EMI from the photo-cathode might be leaking from small apertures in the photo-cathode matching box, which is possibly a weaker source than the bare MCP microstrips. In addition to being higher, the MCP pulse lasted for about twice as long as the photo-cathode pulse. The phosphor pulse and the pulse of the electromagnets had a negligible effect. More tests need to be performed, especially since the PMT was not as well sealed against EMI as it is in NIF.

We concluded from the measurements that DIXI needed additional shielding to lower the diagnostic-generated external fields, so a "broadcast" shield was designed. The broadcast shield will simultaneously shield the external environment from diagnostic-generate fields (mainly from the MCP and phosphor), as well as partially shield the DIXI CCD and cables from diagnostic-generate fields.

The broadcast shield will only partially shield the DIXI CCD and cables because the CCD will still be open at the front, allowing fields to enter. As in the case of ARIANE, keeping the front of the DIXI CCD open is necessary to allow light from the phosphor to enter. Again, if this becomes a problem, transparent EMI films could be investigated as a possible solution.

An additional possible issue is the metal tube in the broadcast shield. It might act as a waveguide and guide the above-cutoff fields from the MCP area of the microstrip to the front of the CCD. Thorough measurements and operational testing after the broadcast shield is installed are planned to determine whether the tube creates the problems mentioned above. The broadcast shield design is sufficiently modular to allow the omission of the metal tube, if that is deemed necessary from the measurements.

Further, we will test whether the broadcast shield lowers the external fields and the effects on the FFLEX PMT. The PMT will be tested in a more realistic scenario, with shielding intact as in NIF. Additional low-frequency magnetic field measurements will be conducted with the solenoid current at the proposed higher operating point to determine if additional magnetic field shielding is required.

\section{CONCLUSIONS AND RECOMMENDATIONS}

Diagnostic-generated EMI is another source of EMI in laser facilities that can cause problems if it is overlooked. High-voltage pulses along microstrips can generate large EMI. The pulsed power and associated cables can also generate EMI. That EMI can affect other diagnostics, as well as the very diagnostic that generated the EMI. Generation of diagnostic-generated EMI has been observed in ARIANE and DIXI. Effects of diagnostic generated EMI have been observed in ARIANE's CCD and on an FFLEX PMT (in the laboratory). Mitigation has been successful in ARIANE, and more tests are planned for DIXI and the FFLEX
PMT. Additional shielding in DIXI is being incorporated earlier in the design cycle in order to protect DIXI and FFLEX from DIXI's diagnostic-generated EMI.

Recommendations for mitigating the effects of diagnosticgenerated EMI include shielding and changing the source, if possible. Sometimes it is not possible to completely shield all of the entry points of vulnerable electronics, as in the case of the front opening of a CCD. Good shielding design practices, coupled with early and realistic testing are essential.
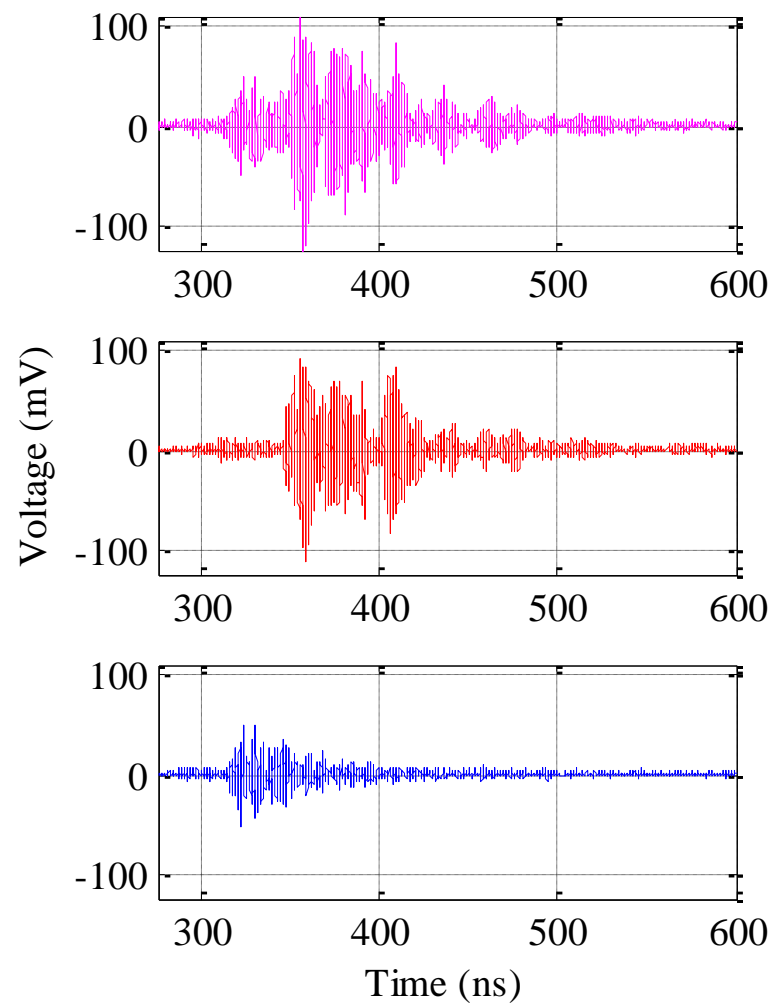

FIG. 4. (color online) Coupled voltage output on nearby FFLEX PMT when DIXI is pulsed in the laboratory. (top) MCP, electromagnets, photo-cathode, and phosphor all pulsed. (middle) Only MCP pulsed. (bottom) Only photo-cathode pulsed.

\section{ACKNOWLEDGMENTS}

This work performed under the auspices of the U.S. Department of Energy by Lawrence Livermore National Laboratory under Contract DE-AC52-07NA27344.

${ }^{1}$ M.J. Mead, D. Neely, J. Gauoin, R. Heathcote, and P. Patel, Rev. Sci. Instrum. 75(10), 4225 (2004)

${ }^{2}$ J. Raimbourg, Rev. Sci. Instrum. 75(10), 4234 (2004).

${ }^{3}$ C. Stoeckl et al., Rev. Sci. Instrum. 77, 10 F506 (2006)

${ }^{4}$ C. G. Brown Jr., E. Bond, T. Clancy, S. Dangi, D. C. Eder, W. Ferguson, J. Kimbrough, and A. Throop, JPCS 244, 032001 (2010).

${ }^{5}$ P. M. Bell et al., Rev. Sci. Instrum. 81 (2010), 10.1063/1.3491208.

${ }^{6}$ T. Hilsabeck et al., Rev. Sci. Instrum. 81, 10E317 (6 pp.) (2010).

${ }^{7}$ E. L. Dewald et al., Rev. Sci. Instrum. 81, 10D938 (2010). 